\title{
Neoadjuvant chemotherapy versus debulking surgery in advanced tubo-ovarian cancers: pooled analysis of individual patient data from the EORTC 55971 and CHORUS trials.
}

\section{DOI:}

10.1016/S1470-2045(18)30566-7

\section{Document Version}

Accepted author manuscript

Link to publication record in Manchester Research Explorer

\section{Citation for published version (APA):}

Jayson, G. (2018). Neoadjuvant chemotherapy versus debulking surgery in advanced tubo-ovarian cancers: pooled analysis of individual patient data from the EORTC 55971 and CHORUS trials. Lancet Oncology, 19(12), 1680-1687. https://doi.org/10.1016/S1470-2045(18)30566-7

\section{Published in:}

Lancet Oncology

\section{Citing this paper}

Please note that where the full-text provided on Manchester Research Explorer is the Author Accepted Manuscript or Proof version this may differ from the final Published version. If citing, it is advised that you check and use the publisher's definitive version.

\section{General rights}

Copyright and moral rights for the publications made accessible in the Research Explorer are retained by the authors and/or other copyright owners and it is a condition of accessing publications that users recognise and abide by the legal requirements associated with these rights.

\section{Takedown policy}

If you believe that this document breaches copyright please refer to the University of Manchester's Takedown Procedures [http://man.ac.uk/04Y6Bo] or contact uml.scholarlycommunications@manchester.ac.uk providing relevant details, so we can investigate your claim.

\section{OPEN ACCESS}




\section{Neoadjuvant chemotherapy versus upfront debulking surgery in advanced tubo-ovarian cancers: meta-analysis of individual patient data from the EORTC and CHORUS trials}

\author{
Ignace Vergote, Corneel Coens, Matthew Nankivell, Gunnar B Kristensen, Mahesh K B Parmar, Tom Ehlen, Gordon C Jayson, Nick Joh Ga \\ Ann Marie Swart, René Verheijen, W Glenn McCluggage, Tim Perren, Pierluigi Benedetti Panici, Gemma Kenter, Antonio Casado 2 \\ Cesar Mendiola, Gavin Stuart, Nick S Reed, Sean Kehoe, and the EORTC and MRC CHORUS study investigators
}

\section{Summary}

Background Individual patient data from two randomised trials comparing neoadjuvant chemotherapy with upfront debulking surgery in advanced tubo-ovarian cancer were analysed to examine long-term outcomes for patients and to identify any preferable therapeutic approaches for subgroup populations.

Methods We did a preplanned meta-analysis of individual patient data from the European Organisation for Research and Treatment of Cancer (EORTC) 55971 trial (NCT00003636) and the Medical Research Council Chemotherapy Or Upfront Surgery (CHORUS) trial (ISRCTN74802813). In the EORTC trial, eligible women had biopsy-proven International Federation of Gynecology and Obstetrics (FIGO) stage IIIC or IV invasive epithelial tubo-ovarian carcinoma. In the CHORUS trial, inclusion criteria were similar to those of the EORTC trial, and women with apparent FIGO stage IIIA and IIIB disease were also eligible. The main aim of the meta-analysis was to show noninferiority in overall survival with neoadjuvant chemotherapy compared with upfront debulking surgery, using the reverse Kaplan-Meier method. Tests for heterogeneity were based on Cochran's Q heterogeneity statistic.

Findings Data for 1220 women were included in the meta-analysis, 670 from the EORTC trial and 550 from the CHORUS trial. 612 women were randomly allocated to receive upfront debulking surgery and 608 to receive neoadjuvant chemotherapy. Median follow-up was 7.6 years (IQR 6.0-9.6; EORTC, 9.2 years [IQR 7.3-10.4]; CHORUS, 5.9 years [IQR 4·3-7·4]). Median age was 63 years (IQR 56-71, range 25-88) and median size of the largest metastatic tumour at diagnosis was $8 \mathrm{~cm}$ (IQR 4.8-13.0, range 0-50). 55 (5\%) women had FIGO stage II-IIIB disease, 831 (68\%) had stage IIIC disease, and $19 \%)$ had stage IV disease, with staging data missing for 104 (9\%) women. [A: please check numbers of women a ] In the entire population, no difference in median overall survival was noted between patients who underwent neoadjuvant chemotherapy and upfront debulking surgerv (27.6 months [IQR 14·1-51.3] and 26.9 months [12.7-50.1], respectively; hazard ratio [HR] 0.97, 95\% CI 0.86- $Q 2 p=0 \cdot 586)$. [A: data in Results were different (I think you had added those for PFS), I've amended to match Results; please check carefully] Median overall survival for EORTC and CHORUS patients was significantly different at 30.2 months (IQR 15.7-53.7) and 23.6 months (10.5-46. spectively (HR 1.20, 95\% CI 1.06-1.36; p=0 004), but was not heterogeneous (Cochran's $Q, p=0 \cdot 17)$. Variable omes were noted in-some cohorts.

Interpretation Long-term follow-up data substantiate previous results showing [A: OK change for "confirrr] that neoadjuy hemotherapy and upfront debulking surgery result in similar overall survival in advanced tubo-ovarian cancer, 1 breferential outcomes [A: can you be specific?] in some [A: which?] patients. This meta-analysis, with long-term follow-up, shows that neoadjuvant chemotherapy is a valuable treatment option for patients with stage IIIC-IV tubo-ovarian cancer, particularly in patients with a high tumour burden at presentation or poor performance status.

Funding National Cancer Institute, Vlaamse Liga tegen kanker (Flemish League against Cancer), Cancer Research

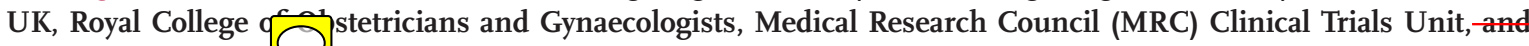
MRC. [A: please che

Copyright (C) 2018 The Author(s) $C$ ished by Elsevier Ltd. This is an Open Access article under the CC BY XXX 4.0 license. [A: please add license ch,

Introduction

[A: we have edited your Article to achieve co 2 ency with Lancet style, avoid repetition, enhance readability, and reduce length. We minimise use of abbreviations] More than $70 \%$ of women with carcinoma of the ovary, fallopian tube, or peritoneum (hereafter referred to as tubo-ovarian cancer) present with advanced disease and usually have a very poor prognosis. ${ }^{1}$ Since Griffiths ${ }^{2}$ reported in 1975 the association between low residual tumour load and improved survival after debulking
Lancet Oncol 2018

Published Online October 30, 2018 http://dx.doi.org/10.1016/ S1470-2045(18)30566-7

See Online/Comment http://dx.doi.org/10.1016/ PII

University Hospitals Leuven, Department of Obstetrics and Gynaecology, Burgium (Prof I Vergote

Department name, European Organisation for Research and Treatment of Cancer, Brussels, Belgium (C Coens MSc); Institute of Clinical Trials and Methodology

(M K B Parmar DPhil), Medical Research Council Clinical Trials Unit at University College London (M Nankivell MSc), London, UK; Norwegian Radium Hospital and Institute for Cancer Genetics and Informatics, Oslo University Hospital, Oslo, Norway (G B Kristensen MD); Department name, University of British Columbia, Vancouver, BC, Canada (T Ehlen MD, Prof G Stuart MD); Department name, Christie Hospital and Department name, University of Manchester, Manchester, UK (Prof G C Jayson PhD); Department name, Royal United Hospitals Bath, Bath, UK (N Johnson MRCOG); Department name, University of East Anglia, Norwich, UK (Prof A M Swart MBBS); Department name, Vrije Universiteit Medical Center, Amsterdam, Netherlands (Prof R Verheijen MD); Department name, Belfast Health and Social Care Trust, Belfast, UK

(Prof W G McCluggage FRCPath); Department name, St James's University Hospital, Leeds, UK (Prof T Perren MD); Department name, University of Rome LaSapienza, Rome, Italy (P B Panici PhD); Center 
Gynaecologic Oncology [A: "Centre for Gynaecological Oncology"?] Amsterdam, University of Amsterdam, Amsterdam, Netherlands (Prof G Kenter MD); Department name, Hospital Universitario

San Carlos, Madrid, Spain (A Casado MD); Department name, Hospital Universitario Doce de Octubre, Madrid, Spain (C Mendiola MD); Beatson Oncology Centre, Glasgow, UK (Prof N S Reed MBBS); and Department name, University of Birmingham, Birmingham, UK (S Kehoe MD)

[A: please ask all authors to check their affiliation is correct and provide their department name (if not already done so)] Correspondence to:

Prof Ignace Vergote, University Hospitals Leuven, Department of

Obstetrics and Gynaecology, Division of Gynaecological Oncology, Leuven Cancer Institute, B-3000 Leuven, Belgium Ignace.vergote@uzleuven.be

\section{Research in context}

\section{Evidence before this study}

Before the start of this analysis on June 6, 2015, and before submission of this Article on March 11, 2018, we searched PubMed with no language restrictions using the keywor ["ovarian cancer"] AND ["randomized" OR "randomized \& do you mean "["randomized" OR "randomised"] or vice versa"?] AND ["clinical trial"] AND ["neoadjuvant chemotherapy" OR "primary chemotherapy"] to identify publications that analysed the role of neoadjuvant chemotherapy followed by interval debulking in comparison with upfront debulking surgery followed by chemotherapy in patients with advanced ovarian cancer. We found that survival data from randomised studies had been published for only two trials: the European Organisation for Research and Treatment of Cancer (EORTC) 55971 trial, and the Medical Research Council Chemotherapy or Upfront Surgery (CHORUS) trial. Both trials concluded that neoadjuvant chemotherapy followed by interval debulking surgery was not inferior to primary debulking surgery followed by chemotherapy in patients with International Federation of Gynecology and Obstetrics (FIGO) stage IIIC or IV ovarian carcinoma. In both studies, no subgroup analyses showed a difference in survival between the two treatment groups. In addition to these two trials, the SCORPION and JCOG0602 randomised trials concluded that perioperative morbidity was better with interval debulking after neoadjuvant chemotherapy than after primary debulking surgery.

\section{Added value of this study}

We report a preplanned individual patient data meta-analysis of the EORTC and CHORUS randomised trials, to examine the long-term outcomes in patients with advanced ovarian cancer treated with neoadjuvant chemotherapy followed by interval debulking versus upfront debulking surgery followed by chemotherapy. We also did subgroup analyses on the basis of stratification factors that were common to both trials-ie, randomising centre, largest tumour size (excluding ovaries) before surgery $(<5 \mathrm{~cm}, \geq 5$ to $\leq 10 \mathrm{~cm},>10 \mathrm{~cm}$ to $\leq 20 \mathrm{~cm}$, or $>20 \mathrm{~cm}$ ), and clinical FIGO stage. Our analysis shows that, with longer median follow-up, both neoadjuvant chemotherapy followed by interval debulking surgery and upfront debulking surgery followed by chemotherapy are associated with similar overall survival and progression-free survival. We also show that neoadjuvant chemotherapy is associated with better progression-free survival and overall survival in women presenting with FIGO stage IV disease, and patients with FIGO stage IIIC disease with extrapelvic metastases smaller than $5 \mathrm{~cm}$ have better progression-free survival after upfront debulking.

\section{Implications of all the available incidence}

[A: can you add a sentence to put your findings in the context of all the available evi ? This section should not simply restate your conclusion have reworded some sections. Please check carefully] The findings of this analysis should be applied in the context of each patient's clinical picture. Participants in this study had FIGO stage IIIC or IV tubo-ovarian cancer with metastatic disease with a high tumour burden at presentation, and many had a poor performance status. This individual patient data meta-analysis suggests that neoadjuvant chemotherapy should be the standard of care for most patients with stage IV tubo-ovarian cancer and that primary surgery should only be undertaken in exceptional circumstances in patients with FIGO stage IV disease on an individual basis. However, women with FIGO stage IIIC disease with extrapelvic metastases smaller than $5 \mathrm{~cm}$ had a significantly better progression-free survival with upfront surgery than with neoadjuvant chemotherapy, so these patients should first be considered for primary debulking surgery. For patients with FIGO stage IIIB or lower disease, there is no evidence to support the use of neoadjuvant chemotherapy instead of primary surgery. surgery, primary surgery has been an essential, or even mandatory, therapeutic strategy in clinical practice. ${ }^{3}$ [A: 40 please check reference numbering, you had referenced Griffiths as number 2 but it was originally 3 in the list] However, to date, no randomised controlled trials have shown that primary debulking surgery improves the prognosis of patients with advanced tubo-ovarian cancer.

An alternative approach to primary debulking surgery is neoadjuvant chemotherapy, administered before attempting cytoreductive surgery. In 2010, the European Organisation for Research and Treatment of Cancer (EORTC) published the first findings from a trial 50 comparing neoadjuvant chemotherapy followed by interval debulking surgery with upfront debulking surgery (EORTC 55971). . This study showed both treatment strategies had similar overall survival and progression-free survival in women with International 55 Federation of Gynecology and Obstetrics (FIGO) stage IIIC or IV tubo-ovarian cancer, and operative and postoperative morbidity was lower with neoadjuvant chemotherapy. These results were later substantiated in the randomised Medical Research Council (MRC) Chemotherapy Or Upfront Surgery (CHORUS) trial, leading to the acceptance of neoadjuvant chemotherapy followed by interval debulking surgery as an alternative to upfront debulking surgery in women with stage IIIC and IV tubo-ovarian cancer. ${ }^{6}$ However, the selection of women with advanced ovarian cancer for neoadjuvant chemotherapy or upfront debulking surgery remains controversial.

In 2003, while accrual for the EORTC study was ongoing but before the start of the CHORUS trial, the EORTC and MRC planned the current analysis with the aim of analysing long-term follow-up of both trials and identifying any subgroups of women that might benefit from neoadjuvant chemotherapy compared with upfront debulking surgery. Herein, we report the results of this analysis. 


\section{Methods}

Study design and data collection

We did a preplanned meta-analysis of individual patient data from the EORTC 55971 trial $^{4}$ and the MRC CHORUS trial, ${ }^{5}$ according to PRISMA guidelines (figure 1; appendix 5 pp 117-120). ${ }^{8}$ Eligibility criteria for these trials and their study designs have been reported elsewhere.,5 The appendix includes the protocols of the EORTC (pp 11-69) and CHORUS (pp 70-116) trials. A list of recruiting sites and investigators is also in the appendix (pp 121-123).

Briefly, in the EORTC trial, ${ }^{4}$ women were eligible if they had had biopsy-proven FIGO stage IIIC or IV invasive epithelial ovarian, primary peritoneal, or fallopian tube carcinoma. If a biopsy specimen was not available, fine-needle aspiration showing an adeno- 15 carcinoma was acceptable under the following conditions: presence of a pelvic adnexal mass, presence of extrapelvic metastases of $2 \mathrm{~cm}$ or larger (measured during diagnostic laparoscopy or laparotomy, or if laparoscopy or laparotomy was not done, on the basis on CT findings), 20 and a ratio of cancer antigen 125 (CA125) to carcinoembryonic antigen (CEA) greater than 25; biopsy findings were mandatory for inclusion in the trial if any of these three criteria was not present. If the CA125:CEA ratio was less than 25 , investigations to exclude a 25 gastrointestinal carcinoma were necessary before entry. In the CHORUS trial, ${ }^{5}$ inclusion criteria were similar to those in the EORTC trial, and women with apparent FIGO stage IIIA and IIIB were also eligible. The size of the largest metastasis was estimated on the basis of CT 30 imaging only in the CHORUS trial.

In both trials, participants were randomly allocated to receive either upfront debulking surgery followed by at least six courses of platinum-based chemotherapy, or three courses of neoadjuvant platinum-based chemo- 35 therapy followed by interval debulking surgery followed by at least three additional courses of platinum-based chemotherapy. ${ }^{4,5}$ In women allocated to receive upfront debulking surgery whose surgery was completed without optimum cytoreduction, interval debulking surgery was 40 permitted, and these patients were incl for analyses in the upfront debulking surgery groun the EORTC trial, [A: addition correct?] randomisation used a minimisation technique and was stratified by the following factors: institution, method of biopsy (imageguided, laparoscopy, laparotomy, or fine-needle aspiration), FIGO stage IIIC or IV disease, and largest tumour size (excluding ovaries) before $r$ ry $(<5 \mathrm{~cm}$, $\geq 5$ to $\leq 10 \mathrm{~cm},>10$ to $\leq 20 \mathrm{~cm}$, or $>20 \mathrm{~cm}$ ). Inrre CHORUS trial, [A: addition correct?] randomisation used a 50 minimisation method with a random element, which stratified patients according to randomising centre, largest radiological tumour size, clinical FIGO stage, and prespecified chemotherapy regimen $[\mathrm{A}$ : this sentence seems like repetition, unless the first sentence (S) bout 55 the EORTC trial and this sentence is about $\mathrm{CH}$ S (as I have edited, by assumption). Can you please

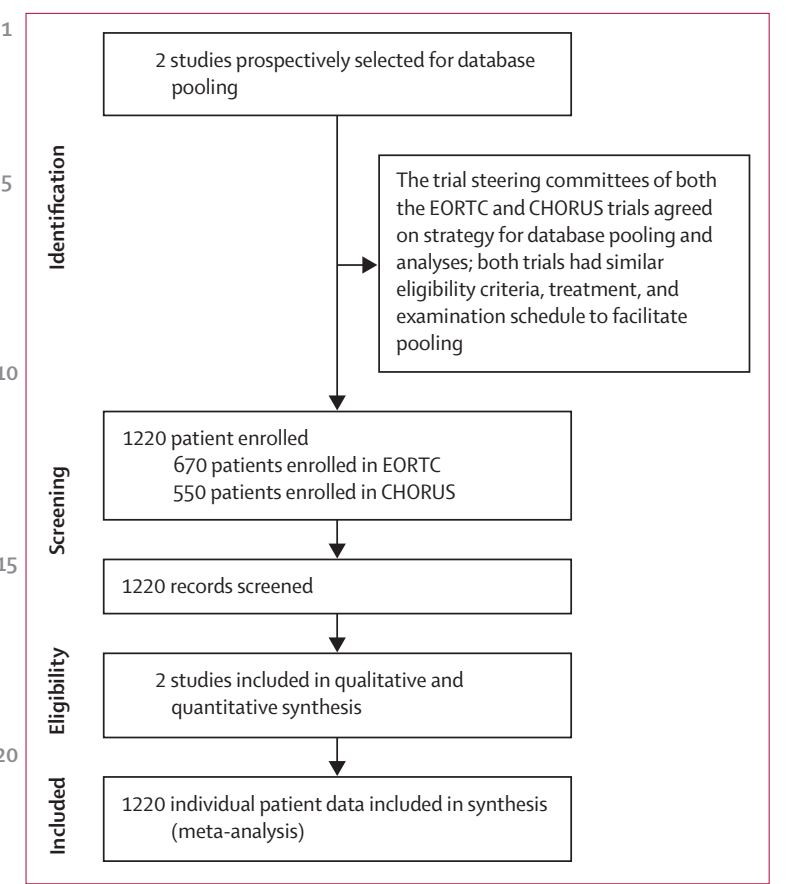

See Online for appendix

Figure 1: PRISMA flow diagram

EORTC=European Organisation for Research and Treatment of Cancer 55971

trial. CHORUS=Medical Research Council Chemotherapy Or Upfront Surgery trial.

\begin{tabular}{llcc|}
\hline & EORTC $(\mathbf{n = 6 7 0 )}$ & CHORUS $(\mathbf{n = 5 5 0 )}$ & Total $(\mathbf{n = 1 2 2 0})$ \\
\hline Age (years) & $62(54-69)$ & $65(58-72)$ & $63(56-71)$ \\
Range & $25-86$ & $26-88$ & $25-88$ \\
Largest metastatic tumour size $(\mathrm{mm})$ & $80(42-140)$ & $80(50-120)$ & $80(48-130)$ \\
(range) &
\end{tabular}

double-check the wording?]. For details on size of residual tumour, residual tumour per country, type of surgery, number of cycles and type of chemotherapy, and time to (re)initiation of chemotherapy, we refer to the original reports. ${ }^{4,5}$

\section{Procedures}

Our analysis was designed in 2003 by the chief investigators of the EORTC and CHORUS trials (IV and $\mathrm{SK})$ and members of the EORTC and MRC trial managing committees. Trial databases were set up to ensure appropriate comparable information was gathered in both trials to allow the planned individual patient data meta-analysis. Women were followed up until database lock. Data from CHORUS were capferred to EORTC headquarters [A: please add adaress] and 


\begin{tabular}{|lccc|}
\hline & $\begin{array}{l}\text { Upfront debulking } \\
\text { surgery }(\mathbf{n}=612)\end{array}$ & $\begin{array}{l}\text { Neoadjuvant } \\
\text { chemotherapy } \\
(\mathbf{n}=608)\end{array}$ & Total $(\mathbf{n}=\mathbf{1 2 2 0})$ \\
\hline Age (years) & $63(55-71)$ & $64(57-70)$ & $63(56-71)$ \\
Range & $25-87$ & $33-88$ & $25-88$ \\
Largest metastatic tumour size $(\mathrm{mm})$ & $80(49-130)$ & $80(47-125)$ & $80(48-130)$ \\
Range & $0-430$ & $0-500$ & $0-500$ \\
CA125 at entry (kU/L) & $1039(409-2548)$ & $1137(446-2606)$ & $1089(431-2599)$ \\
Range & $16-39323$ & $15-41456$ & $15-41456$ \\
Data are median (IQR), unless otherwise indicated. CA125=cancer antigen 125. & \\
\hline Table 2: Baseline characteristics, by allocated treatment & & \\
\hline
\end{tabular}

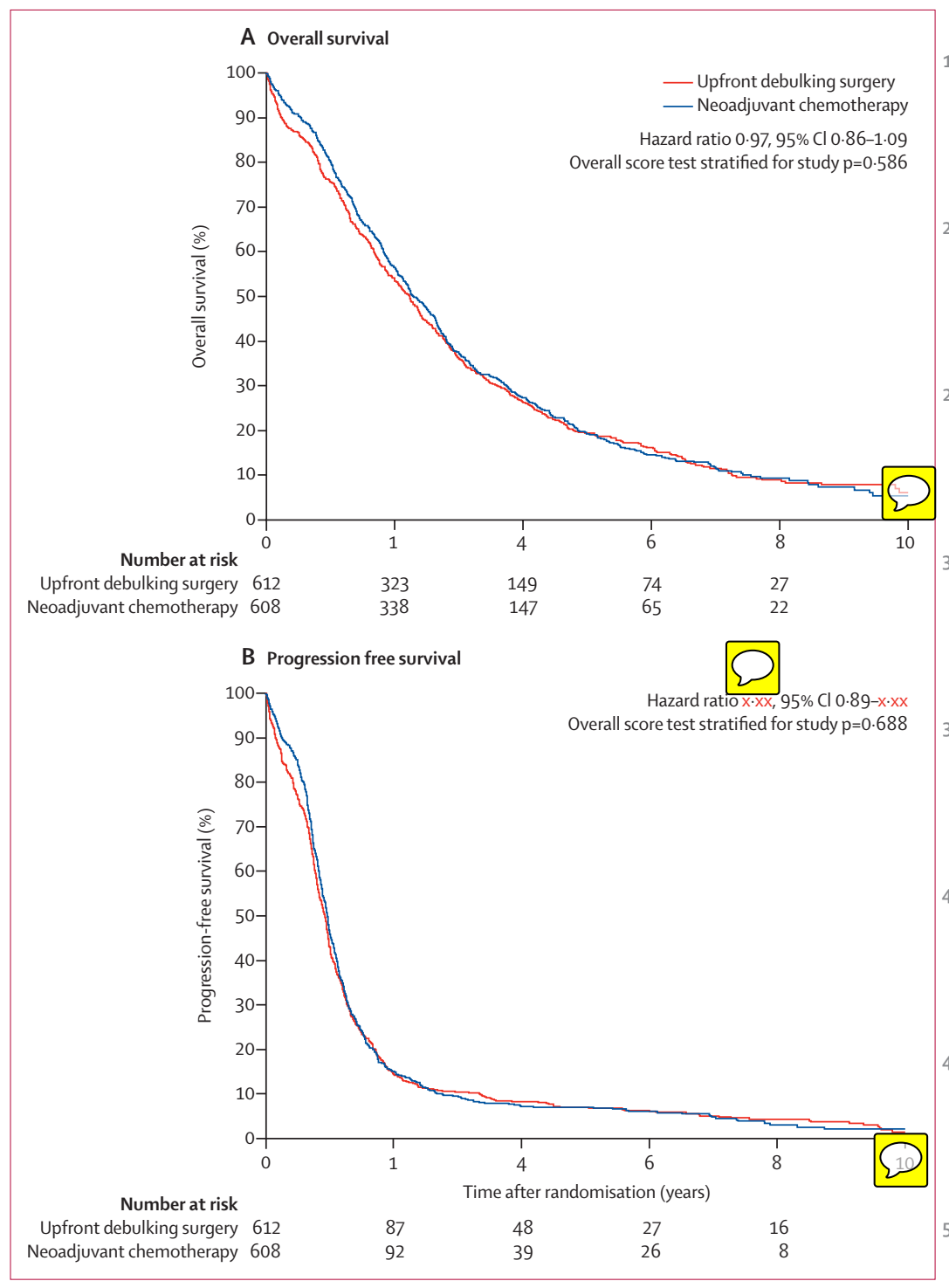

Figure 2: Overall survival and progression-free survival, bytroatment

[A: please provide HR and 95\% Cl for each plot; numbers _ist t 10 years are missing, please add]
1 Kaplan-Meier method and calculates time-to-events for all patients; in the original CHORUper, however, only the median duration of follow for surviving patients was calculated. [A: Ok as edited?]

5 The primary outcome of our meta-analysis was overall survival, and the prespecified secondary endpoint was progression-free survival. Prespecified subgroup analyses were done on the basis o stratification factors that were common to both trials: randomising centre, 10 largest tumour size (excluding ovaries) before surgery $(<5 \mathrm{~cm}, \geq 5$ to $\leq 10 \mathrm{~cm},>10$ to $\leq 20 \mathrm{~cm}$, or $>20 \mathrm{~cm})$, and clinical FIGO stage. Definitions for overall survival and progression-free survival have been published elsewhere. ${ }^{4}$

15

\section{Statistical analysis}

At the planning stage, we estimated that the pooled dataset would contain between 800 and 900 events (deaths). Assuming a median overall survival of 3 years, 20 this number of events allowed assessment of noninferiority, ${ }^{9,10}$ with a one-sided type I error of 0.05 and a power of $80 \%$, with inferiority regarded as an increase of more than $18-19 \%$ in hazard. Similarly, the atimated number of events would allow $90 \%$ power in uding a 25 hazard increase of 22-23\%. [A: text in red added to explain "it" and "this"] Applying a two-sided test of superiority at $5 \%$, the dataset would allow detection of an $18 \%$ increase in hazard with $80 \%$ power.

The principal analysis was done on an intention-to30 treat basis. Median overall survival and progression-free survival were estimated by the Kaplan-Meier method and compared via the log-2 test. Hazard ratio (HR) estimates and their 95\% CIs were obtained from a Cox proportional hazards model. In those subgroups for 35 which the proportional hazards assumption was violated, restricted mean survival times were al plated to provide a more useful [A: more useful trarr what?] general measure to report the average survival times between the two treatment arms. ${ }^{11}$ Multivariable time-to-event 40 analyses were done using a Cox proportional hazards model, with univariate screening followed by a multivariable stepwise selection procedure. ${ }^{12}$ All baseline characteristics and results were checked for homogeneity between the two studies and stratified per trial when 45 possible. Tests for heterogeneity in progression-free survival or overall survival were based on Cochran's Q heterogeneity statistic. All analyses were done with SAS, version 9.4.

\section{5ole of funding source}

The funders of the studies had no role in study design, data collection, data analysis, data interpretation, or writing of the report. CC, MN, and MKBP had full access to MRC CHORUS raw data. CC had full access to EORTC analysed by us [A: who?] in cooperation with the EORTC 5555971 raw data. The corresponding author had full access statistician (CC). We derived median follow-up with the EORTC standard method, which uses the reverse to all data in the study and had final responsibility for the decision to submit for publication. 


\section{Results}

Patients' data from both trials were updated and merged into one database (database lock for EORTC was on June 6, 2015; for CHORUS, June 3, 2014), which contained 1220 patients with tubo-ovarian cancer who had been randomly allocated to receive either upfront debulking surgery $(\mathrm{n}=612)$ or neoadjuvant chemotherapy $(\mathrm{n}=608)$. Total combined recruitment lasted almost 12 years (in EORTC, 670 patients from Oct 12, 1998 to Nov 29, 2006; in CHORUS, 550 patients from 10 March 5, 2004, to Aug 26, 2010). Median follow-up was $7 \cdot 6$ years (IQR $6 \cdot 0-9.6$ years; EORTC, $9 \cdot 2$ years [IQR 7.3-10.4]; CHORUS, 5.9 years [IQR 4.3-7.4]). Characteristics of patients by study and study group are summarised in tables 1 and $\mathbf{2}$, respectively. Pretreatment 15 characteristics were well balanced between both treatment groups. Median age was 63 years (IQR 56-71, range $25-88$ ) and median size of the largest metastatic tumour at diagnosis was $8 \mathrm{~cm}$ (IQR $4 \cdot 8-13 \cdot 0$, range 0-50). 55 (5\%) women had FIGO stage II-IIIB disease, 2 $831(68 \%)$ had stage IIIC disease, and 230 (19\%) had stage IV disease, with staging data missing f 194 (9\%) women. [A: I've added these data from the S $D$ hary, to ensure the Results contain all data in the Summary]

Overall survival and progression-free survival for the 2 entire population were similar with neoadjuvant chemotherapy and upfront debulking surgery, with median overall survival of 27.6 months (IQR 14.1-51.3) and 26.9 months $(12 \cdot 7-50 \cdot 1)$, respectively (HR 0.97 , 95\% CI 0.86-1.09; $\mathrm{p}=0 \cdot 568)$, [A: yo 2$]$ w written in the 30 next sentence (now deleted) 0.87 ; please check] and progression-free survival of 11.6 months (IRD $79-17.7)$ and $11 \cdot 1$ months (6.4-17.5), respectively (H $95 \% \mathrm{CI}$ 0.89-xx; $p=0 \cdot 688$; figure 2). [A: please add HR and 95\% CI] The lower one-sided 95\% CIs for overall survival and 35 progression-free survival excluded the $18 \%$ noninferiority margin.

Overall survival was significantly better in the EORTC trial as compared with the CHORUS trial (median 30.2 months [IQR 15.7-53.7] vs 23.6 months 40 [10.5-46.9]; HR 1.20, 95\% CI 1.06-1.36; $\mathrm{p}=0 \cdot 004$; figure 3), but progression-free survival was similar in the two trials (median 11.5 months [IQR 8.0-17.0] vs 10.9 months [6.1-18.1]; HR 0.96, 95\% $0 \cdot 86-1 \cdot 08$; $\mathrm{p}=0.531$; appendix p 1). [A: I've deleted $\bigcirc$ rst page of your appendix because we add a title page later; please check $p$ values in appendix, log-rank is $p=0.530$ ] Overall survival and progression-free survival according to trial and treatment groups are prese $\rightarrow$ in the appendix (pp 2, 3). [A: please check p value 2 ppendix, log-rank $\mathrm{p}$ differs in plot and table below plot] Cochran's $\mathrm{Q}$ for heterogeneity was not significant for either overall survival $(p=0 \cdot 17)$ or progression-free survival $(p=0 \cdot 32)$.

Median overall survival was significantly different for women with FIGO stage IV disease compared with those 5 with stage III and II cancer (median 23.3 months [IQR $12 \cdot 4-40 \cdot 8]$ vs $30 \cdot 0$ months $[15 \cdot 6-55 \cdot 7]$ and

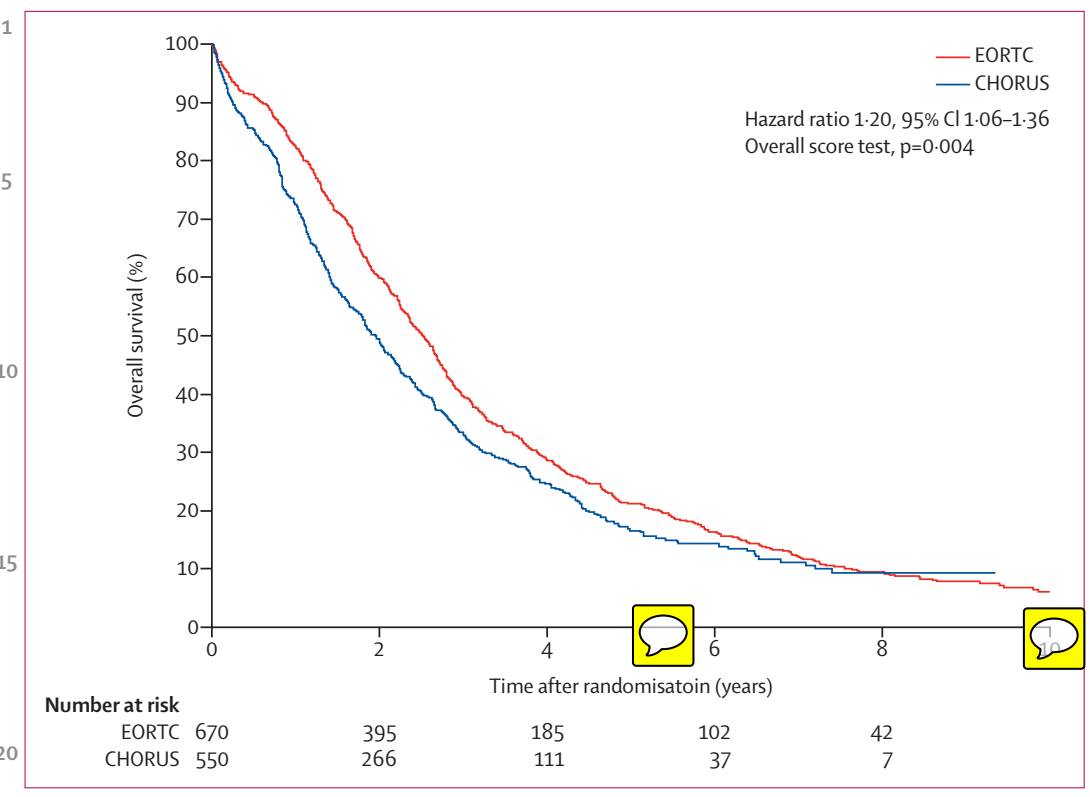

Figure 3: Overall survival, by study

EORTC=European Organisation for Research and Treatment of Cancer 55 al. CHORUS=Medical Research Council Chemotherapy Or Upfront Surgery trial. [A: please provide HR a $6 \mathrm{Cl}$; numbers at risk at 10 years are missing, please add]

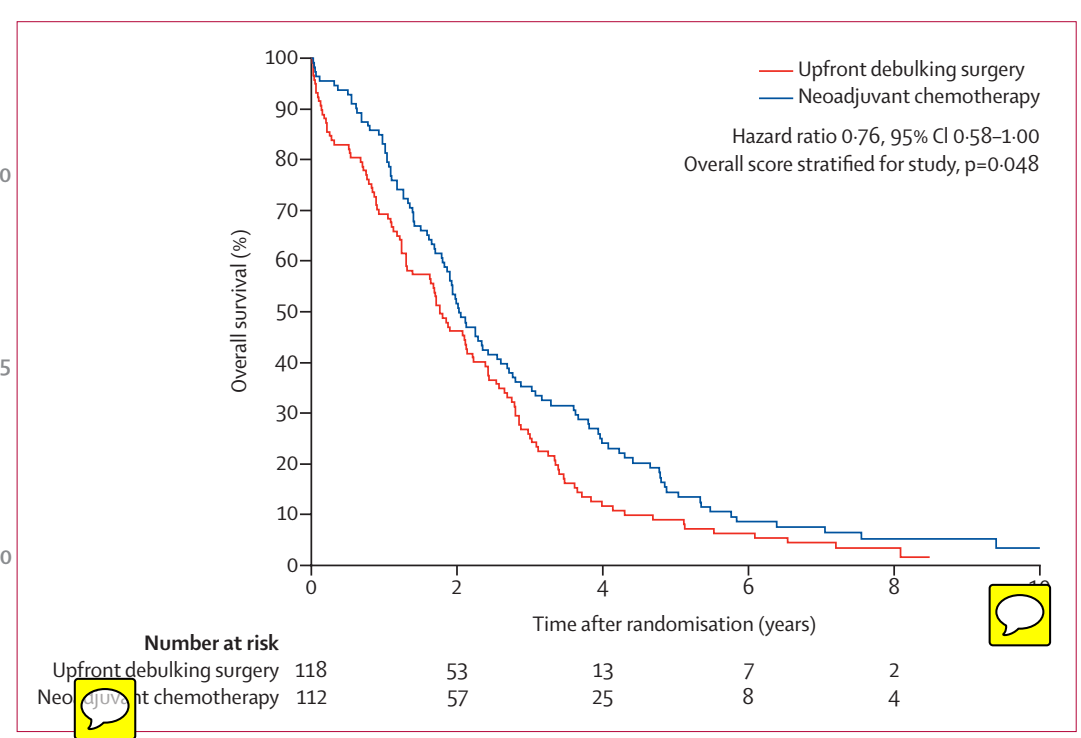

5 Figure 4: Overall survival in patients with FIGO stage IV disease, by treatment

$\mathrm{FIGO}=$ International Federation of Gynecology and Obstetrics. [A: numbers at risk at 10 years are missing, please add]

45.4 months [31.6IV vs stage II, HR 2.75, 95\% CI 1.49-5.08; for stage III vs stage II, 1.92, $\$ 2$ CI 1.05-3.49; p<0.0001 frrtrend [A: OK? Is the p value across all groups?]; apper \&p 4). [A: please check edits in red are correct; data match appendix, but original text was the other way around] Overall survival was similar with neoadjuvant chemotherapy and upfront debulking surgery in patients with stage IIIC disease (respectively, median 30.8 months [IQR 16.5-51.3] and 


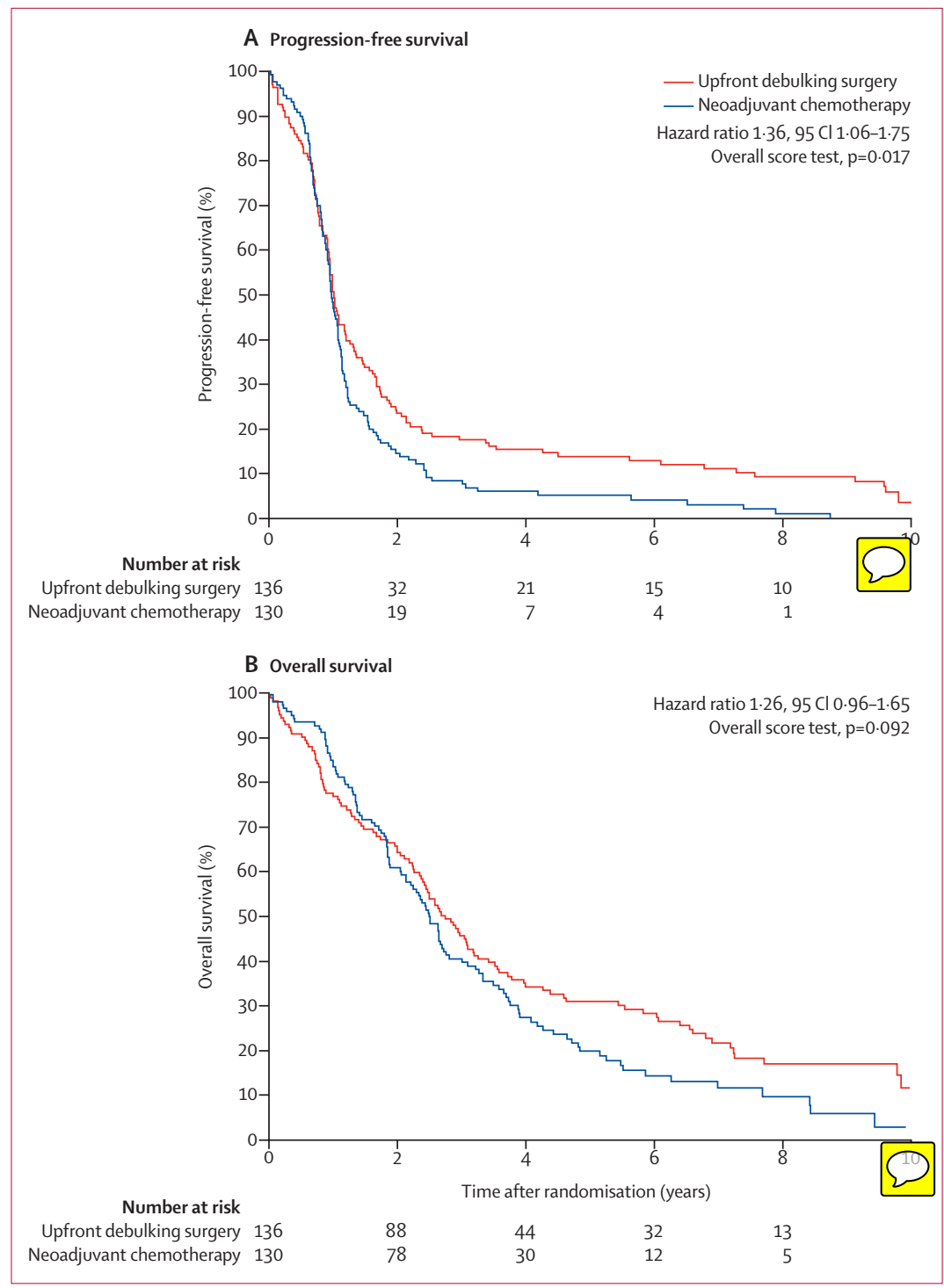

Figure 5: Progression-free survival and overall survival in patients with FIGO stage IIIC disease and a largest metastatic tumour size less than $5 \mathrm{~cm}$ at entry, by treatment

FIGO=International Federation of Gynecology and Obstetrics. [A: numbers at risk at 10 years are missing, please add]

$128 \cdot 4$ months $[14 \cdot 1-55 \cdot 7]$; HR $1 \cdot 04,95 \%$ CI $0 \cdot 90-1 \cdot 21$; $\mathrm{p}=0.569$; appendix $\mathrm{p} 5$ ). Progression-free survival was also similar with neoadjuvant chemotherapy and upfront debulking surgery in patients with stage IIIC disease

5 (median $12 \cdot 2$ months [IQR 8.4-18.3] and 11.7 months [7.5-19 espectively; HR $1 \cdot 06,95 \%$ CI $0 \cdot 92-1 \cdot 22$ $\mathrm{p}=0.422$ pendix p 6). [A: please chack p values in appendix, log-rank is $\mathrm{p}=0$. 428] $]$ n patients with stage IV tubo-ovarian cancer, neoadjuvant chemotherap

10 was associated with significantly better overall surviva than was upfront debulking surgery (median 24.3 months [IQR 14.1-47.6] vs 21.2 months [10.0-36.4]; HR 0.76, 95\% CI 0.58-1.00; $\mathrm{p}=0 \cdot 048$; figure 4). [A: since you do not mention figure 4B in the Results, I've deleted it] Forest 15 plots for overall survival according to FIGO stage are in the appendix $\bigcirc$ [A: if you wan 2 nclude figure 4B, please add it 2 e appendix] Progression-free survival was alse significantly better in stage IV disease with neoadjuvant chemotherapy than with upfront debulking 20 surgery (median 10.6 months [IQR 7.9-15.0] vs 9.7 months [5.2-13.2], eSpectively; HP 7 , 95\% CI $0 \cdot 59-1 \cdot 00 ; \mathrm{p}=0 \cdot 048 ;$ apperux $\mathrm{p}$ 7). [A: $\Omega$ se check $\mathrm{p}$ values in appendix, $\mathrm{p}=0.049$ on plot]

Patients with extrapelvic metastases smaller than $5 \mathrm{~cm}$ 25 at the time of randomisation had the best overall survival of all participants (appendix p 8). In patients with FIGO stage IIIC disease and a largest metastatic tumour size less than $5 \mathrm{~cm}$, progression-free survival was better with upfront debulking surgery than with neoadjuvant 30 chemotherapy (median $12 \cdot 2$ months [IQR $8 \cdot 5-23 \cdot 3$ ] vs 11.7 months [8.3-16.4]; HR 1.36 , 95\% CI $1.06-1 \cdot 75$ $\mathrm{p}=0.017$; figure $5 \mathrm{~A}$ ) Forest plots according to largest metastatic tumour size are in the appendix (p 9). Overall survival did not differ between upfront debulking surgery 35 and neoadjuvant chemotherapy (median 33.0 months [IQR 13.5-78.7] and 30.2 months [16.5-51.3], respectively; HR 1.26, 95\% CI 0.96-1.65; p=0.092; figure $5 \mathrm{~B}$ ). Because of deviation from the proportional hazards assumption in this subgroup, restricted mean 40 survival times are presented in table 3. Age and performance status were not predictive for treatment effect on survival (appendix p 10)

\section{Discussion}

Restricted mean $\quad 95 \% \mathrm{Cl}$ survival time (months)

Overall surviva

Upfront debulking surgery $\quad 47 \cdot 3 \quad 40 \cdot 4-54 \cdot 1$

Neoadjuvant chemotherapy $\quad 39 \cdot 3$

Progression free survival

Upfront debulking surgery

$27 \cdot 5$

$33 \cdot 9-44 \cdot 8$

Neoadjuvant chemotherapy $\quad 17 \cdot 0$

$21 \cdot 2-33 \cdot 8$

$13 \cdot 8-20 \cdot 2$

$\mathrm{FIGO}=$ International Federation of Gynecology and Obstetrics

Table 3: Estimates of restricted mean survival time in patients with FIGO stage IIIC disease and largest metastatic tumour size less than $5 \mathrm{~cm}$ at study entry
45 This preplanned analysis of updated data from the EORTC and CHORUS trials assessing neoadjuvant chemotherapy versus upfront debulking surgery in advanced tubo-ovarian cancer (stage IIIC and IV) shows that, with long-term follow-up, neoadjuvant chemo50 therapy results in non-inferior overall survival and progression-free survival as compared with upfront debulking surgery. The planned non-inferiority marginan increase in 0 more than $18-10 \%$ was well outside the lowe 2 inds of the 95\% CIs 110 and 13\% 55 [A: check against Results, I queried 95\% CIs] for progression-free survival and overall survival, respectively). Hence, this meta-analysis with long-term 
follow-up substantiated previous findings showing that 1 both upfront debulking surgery and neoadjuvant chemotherapy are potential treatment options for patients with FIGO stage IIIC or IV tubo-ovarian cancer. The analysis also showed that patients diagnosed with stage IV disease had significantly better progression-free survival and overall survival with neoadjuvant chemotherapy as compared with upfront debulking surgery, whereas women with stage IIIC disease with extrapelvic metastases smaller than $5 \mathrm{~cm}$ had significantly better progression-free survival with upfront debulking surgery as compared with neoadjuvant chemotherapy. For women with stage III disease and larger metastases $(\geq 5 \mathrm{~cm})$, either approach resulted in the same overall survival. Our analyses showed that in women with stage IIIC disease and extrapelvic metastases at diagnosis smaller than $5 \mathrm{~cm}$, survival curves for both progressionfree survival and overall survival cross in both treatm groups [A: OK change for "both progression-free surv 2 and overall survival curves have crossing treatment 20 arms" to avoid use of "arms" which is not Lancet style?], indicating deviation from the proportional hazards assumptions. Therefore, restricted mean survival times give a better indication of the treatment effect than do median times. ${ }^{11}$ These findings indicate that when 25 deciding on a treatment strategy, one should account not only for the risk of perioperative morbidity ${ }^{6}$ and the possibility of debulking the patient's disease to zero residual tumour ${ }^{7}$ but also for FIGO stage and the extent of metastatic disease at presentation.

Although both the EORTC and CHORUS trials permitted cytological diagnosis of malignant disease, the evolution in knowledge regarding subtypes of tuboovarian cancer disease means that only histology can reliably distinguish between high-grade and low-s serous carcinomas at the time of writing. ${ }^{13}$ This rel 2 on histological diagnosis [A: OK addition to clarify meaning of "this"?] is important because low grade serous carcinomas are ${ }^{\mathrm{h}}$ less sensitive to chemotherapeutic regimens 2 an which other tumour 40 types? Than other treatment strategies? Please add comparator] and primary surgery is an important and much preferred-intervention in this group [A: preferable to what? Please add comparator] [A: How d his management preference relate to reliance on his 2 ical diagnosis (if that is what you meant by "this"? Please clarify meaning of this sentence]. ${ }^{14}$ Therefore, to facilitate optimum decision making, tissue should be obtained for histological diag@s in all cases of tubo-ovarian cancer [A: correct addition?] and assessed in combination with extensive radiological imaging. Obtaining tissue for histological examination is usually possible by use of image-guided biopsy (usually of the omental cake), although a laparoscopic approach is necessary in some cases and provides additional information on disease distribution, which can be included in the decisionmaking process..$^{15-17}$
Both the EORTC and CHORUS trials investigated the timing of surgery in advanced tubo-ovarian cancer these trials have been criticised [A: can you refermece this criticism?] for inclusion of low proportions of patients with no macroscopic disease (R0) and for $\mathrm{P}$ survival [A: OK as edited, since Lancet style is to use of "rates" where there is no time component?]. However, at the time the patients in trials were enrolled [A: OK change for "randomised"?], neoadjuvant chemotherapy was not accepted as an alternative to upfront debulking surgery, and furthermore most patients recruited to these trials had extensive FIGO stage IIIC or IV disease that was visible on CT. Moreover, the SCORPION 15 and ICOGO60218 randomised trials [A what was the date of these trials? What interventions were they testing, in what population?] concluded that interval debulking after neoadjuvant chemotherapy was associated with improved perioperative morbidity as compared with primary debullking surgery. A randomised trial of neoadjuvant chemotherapy versus primary debulking surgery in advanced tubo-ovarian cancer (the TRUST trial; NCT /ISRCTNAX] [A: can you reference or provide an NCT id or other registry id?], has recuited up to $50 \%$ of patients with maximal e (RO) [A: OK change for " pationts from selected centres have been recruited with R0 of $50 \%$ or more"?. The results of this trial are awaited.

One limitation of our meta-analysis is that only patients with FIGO stage IIIC and IV disease were included in 30 the EORTC trial, whereas in the CHORUS trial only a few patients with stage IIIA and B were included. Furthermore, the number of patients with stage IIIC-IV disease without residual tumour after upfront debulking surgery was lower in the CHORUS trial than in the 5 EORTC trial.

Application of the findings of this analysis to the care of women with FIGO stage IIIC or IV tubo-ovarian cancer should be assessed in the context of each patient's clinical picture. Women in the EORTC and CHORUS studies that contributed data to this analysis had metastatic disease with a high tumour burden at presentation, and many had a poor performance status. ${ }^{19}$ This clinical scenario is not uncommon, and improving outcomes for this population is as important-if not more so-than for patients with better prognostic factors. The results of this analysis are derived from one of the largest cohorts of women with FIGO stage IV disease in phase 3 studies. Although some patients with stage IV disease have a better prognosis and present with less spread and more easily resectable disease ${ }^{14}$ than other patients with stage IV disease [A: this seems rather obvious; an you explain what you mean by inclusion of this sentence?], eur data suggest that neoadjuvant chemotherapy should be the standard of care for most patients with FIGO stage IV tubo-ovarian cancer, and primary surgery should only be undertaken in exceptional circumstances in women selected on an individual basis. 


\section{Contributors}

All authors contributed to study design and study implementation. IV and SK wrote the draft of the report, and all authors were involved in writing and approval of the report. All authors have seen and approved the final version and, after consultation with the collaborators, agreed to submit for publication.

\section{Declaration of interests}

MN reports grants from the Medical Research Council Clinical Trials Unit and Cancer Research UK, during the conduct of the study. N reports that the Royal United Hospital (his employing institution) received support [A: from EORTC?] for a clinical trials nurse, who obtained and eril data from some participants in one of the trials reported her 2 hich?]. CM reports personal fees an trat expenses from Roche Farma España, outside the submitted work 2 expenses from AstraZeneca, outside the submitted work; and other [A: please say what "other" was] from Pharmamar, outside the submitted work. TP reports personal fees and non-financial support from AstraZeneca, outside the submitted work; non-financial support from Roche and IGEA Medical, outside the submitted work; and is co-chief investigator for the ICON7 trial of bevacizumab in first-line treatment of patients with advanced ovarian cancer. IV, CC, GBK, MKBP, TE, GCJ, AMS, RV, WGMcC, PBP, GK, AC, GS, NSR, and SK declare no competing interests.

\section{Acknowledgments}

This study was supported by grants (2U10 CA11488-28 through 2U10 CA011488-36) from the National Cancer Institute; and by a donation from the Vlaamse Liga tegen kanker (Flemish League against Cancer) to the EORTC Charitable Trust. Funding was also provided by Cancer Research UK. Funding for a pilot phase of the trial was provided by the Royal College of Obstetricians and Gynaecologists and was supported by 25 core Medical Research Council (MRC) Clinical Trials Unit (CTU) funding. The MRC was the trial sponsor, and the trial was un rtan and analysed at the MRC CTU. [A: can you please be clearer a organisations sponsored which trial(s)? ie, EORTC, CHORUS, and this meta-analysis?]

\section{References}

1 Ferlay J, Steliarova-Foucher E, Lortet-Tieulent J, et al. Cancer incidence and mortality patterns in Europe: estimates for 40 countries in 2012. Eur J Cancer 2013; 49: 1347-403.

2 Griffiths CT. Surgical resection of tumor bulk in the primary treatment of ovarian carcinoma. J Natl Cancer Inst Monogr 1975 42: 101-04.

3 Berek J, Tropé C, Vergote I. Surgery during chemotherapy and at relapse of ovarian cancer. Ann Oncol 1999; 10: 3-7.

4 Vergote I, Tropé CG, Amant F, et al. Neoadjuvant chemotherapy or primary surgery in stage IIIC or IV ovarian cancer. N Engl J Med 2010; 363: 943-53.

5 Kehoe S, Hook J, Nankivell M, et al. Primary chemotherapy versus primary surgery for newly diagnosed advanced ovarian cancer (CHORUS): an open-label, randomised, controlled, non-inferiority trial. Lancet 2015; 386: 249-57. 30
16 Wright AA, Bohlke K, Armstrong DK, et al Neoadjuvant chemotherapy for newly diagnosed, advanced ovarian cancer: Society of Gynecologic Oncology and American Society of Clinical Oncology clinical practice guideline. J Clin Oncol 2016; 34: $3460-73$.

57 Vergote I, du Bois A, Amant F, Heitz F, Leunen K, Harter P. Neoadjuvant chemotherapy in advanced ovarian cancer: on what do we agree and disagree? Gynecol Oncol 2013; 128: 6-11.

8 Moher D, Liberati A, Tetzlaff J, Altman DG. Preferred reporting items for systematic reviews and meta-analyses: the PRISMA statement. PLoS Med 2009; 6: e1000097.

9 Piaggio G, Elbourne DR, Altman DG, Pocock SJ, Evans SJ.

10 Reporting of noninferiority and equivalence randomized trials: an extension of the CONSORT statement. JAMA 2006; 295: 1152-60.

10 Mauri L, D'Agostino RB Sr. Challenges in the design and interpretation of noninferiority trials. N Engl J Med 2017; 377: 1357-67.

11 Royston P, Parmar MK. The use of restricted mean survival time to estimate the treatment effect in randomized clinical trials when the proportional hazards assumption is in doubt. Stat Med 2011; 30: 2409-21.

12 Collett D. Strategy for model selection. In: Collett D, ed. Modelling survival data in medical research. London: Chapman and Hall, 1994: 78-83.

2013 Grabowski JP, Harter P, Heitz F, et al. Operability and chemotherapy responsiveness in advanced low-grade serous ovarian cancer: an analysis of the AGO Study Group metadatabase. Gynecol Oncol 2016; 140: 457-62.

14 Gockley A, Melamed A, Bregar AJ, et al. Outcomes of women with high-grade and low-grade advanced-stage serous epithelial ovarian cancer. Obstet Gynecol 2017; 129: 439-47.

15 Fagotti A, Ferrandina G, Vizzielli G, et al. Phase III randomised clinical trial comparing primary surgery versus neoadjuvant chemotherapy in advanced epithelial ovarian cancer with high tumour load (SCORPION trial): final analysis of perioperative outcome. Eur J Cancer 2016; 59: 22-33.

16 Rutten MJ, van Meurs HS, van de Vrie R, et al. Laparoscopy to predict the result of primary cytoreductive surgery in patients with advanced ovarian cancer: a randomized controlled trial. J Clin Oncol 2017; 35: 613-21.

17 Vergote IB, van Nieuwenhuysen E, Vanderstichele A. How to select neoadjuvant chemotherapy or primary debulking in patients with stage IIIC or IV ovarian carcinoma. J Clin Oncol 2016; 34: 3827-28.

18 Onda T, Satoh T, Saito T, et al. Comparison of treatment invasiveness between upfront debulking surgery versus interval debulking surgery following neoadjuvant chemotherapy for stage III/IV ovarian, tubal, and peritoneal cancers in a phase II randomised trial. Eur J Cancer 2016; 64: 22-31.

19 Van Meurs HS, Tajik P, Hof MH, et al. Which patients benefit most from primary surgery or neoadjuvant chemotherapy in stage IIIC or IV ovarian cancer? An exploratory analysis of the European Organisation for Research and Treatment of Cancer 55971 randomised trial. Eur J Cancer 2013; 49: 3191-201. 